\title{
Growth and survival of Trichomonas vaginalis
}

\author{
IOANNA KOSTARA, H. CARAGEORGIOU, D. VARONOS and S. TZANNETIS* \\ Departments of Experimental Pharmacology and *Microbiology, School of Medicine, University of Athens, \\ Goudi, Athens 11527, Greece
}

\begin{abstract}
This study confirmed that Feinberg and Whittington medium was suitable for the cultivation and detailed study of the growth cycle of two clinical strains of Trichomonas vaginalis under anaerobic conditions. Both strains showed a similar growth pattern characterised by early but slow growth, extended duration of the logarithmic phase and limited survival never exceeding $144 \mathrm{~h}$. Duration of survival and growth rate were inversely proportional to the inoculum density. Growth rate was pH dependent; $\mathrm{pH}$ values in the range 6.9-6.5 delayed the initiation of growth of $T$. vaginalis for at least $48 \mathrm{~h}$. On the other hand, $\mathrm{pH}$ values of 6.4-4.5 were indifferent or slightly favourable for growth during the logarithmic and survival in the early decline phase. Normal saline and Ringer's solution exerted an early and progressively lethal effect on trichomonads and led to the disappearance of protozoa suspended in them in $150 \mathrm{~min}$. In general, these in-vitro results shed light on some aspects of the biology of $T$. vaginalis and contribute to a better understanding of the epidemiology and clinical manifestations of the infection.
\end{abstract}

\section{Introduction}

The flagellate protozoon Trichomonas vaginalis causes a mild urogenital infection [1-3]. It has a world-wide distribution and its prevalence in developed countries in women and men ranges from 5 to $20 \%$ and $2-10 \%$ respectively. The parasite is mainly transmitted by sexual intercourse and occasionally by direct contact with infected fomites [3]. For all these reasons and because of an association between chronic trichomoniasis and cancer of the cervix in females and infertility in males, the infection has long attracted scientific interest [3-5]. However, knowledge of the survival and growth of $T$. vaginalis under laboratory conditions is inadequate or uncertain because of the difficulties in culturing it.

The present work aimed to determine the dynamics of the growth cycle of two clinical strains of $T$. vaginalis in Feinberg and Whittington medium $[6,7]$ and its survival in two other common suspending media, as such results might contribute to a better understanding of the life-cycle and the infectivity of the parasite under conditions of natural infection.

Received 17 June 1997; revised version accepted 5 Nov. 1997.

Corresponding author: Dr D. I. Kostara, 6 Diomidus St., N. Liossia, Athens 13122, Greece.

\section{Materials and methods}

\section{Strains}

Two clinical strains of $T$. vaginalis isolated from two cases of acute vaginal trichomoniasis were used. Diagnosis of the infection was based on clinical signs and on the presence of motile parasites in wet preparations of vaginal exudate.

\section{Microbiological methods}

Samples were taken with sterile cotton swabs and were transported to the laboratory within $30 \mathrm{~min}$ of sampling. Swabs were vortex mixed for $30 \mathrm{~s}$ in $2 \mathrm{ml}$ of Feinberg and Whittington nutrient medium at $\mathrm{pH}$ 6.4. Inoculated tubes were incubated anaerobically in an Oxoid Anaerobic System at $37^{\circ} \mathrm{C}$ for $72 \mathrm{~h}$. These $72-\mathrm{h}$ cultures were used to prepare the standard inocula for any further growth and survival testing, as preliminary experiments showed that viable counts were comparatively more stable at this phase than at any other. Tubes containing $2.7 \mathrm{ml}$ of Feinberg and Whittington medium, Ringer solution or normal saline were inoculated separately with $0.3 \mathrm{ml}$ of a standard inoculum of each strain and the tubes were incubated anaerobically as described above. Cultures in Feinberg and Whittington liquid medium were sampled at intervals of $0,2,4,6$, $8,24,48,72,96,120$ and $144 \mathrm{~h}$, and growth curves of strains $\mathrm{A}$ and $\mathrm{B}$ were made by calculating the mean viable counts of 36 and 27 successive cultures, respectively. Suspensions of both strains in the two 
diluents adjusted to $\mathrm{pH} 6.4$ with $0.1 \mathrm{M} \mathrm{Na}_{2} \mathrm{HPO}_{4} / 0.1 \mathrm{M}$ $\mathrm{KH}_{2} \mathrm{PO}_{4}$ were sampled every $15 \mathrm{~min}$ and survival curves were made by calculating the mean viable counts in three separate suspensions of each strain.

The effect of $\mathrm{pH}$ on the growth and survival of trichomonads was tested only on strain A in Feinberg and Whittington liquid medium buffered at $\mathrm{pH} 6.9$ with $0.1 \mathrm{M} \mathrm{Na}_{2} \mathrm{HPO}_{4} / 0.1 \mathrm{M} \mathrm{KH}_{2} \mathrm{PO}_{4}$ as well as in the same medium only initially adjusted to $\mathrm{pH} 6.9$ by the addition of $\mathrm{N} / 10 \mathrm{NaOH}$. Growth curves were made by calculating the mean viable counts of three successive cultures in each type of medium under the conditions used throughout this study. Populations of motile trichomonads of each strain in cultures and in suspensions were determined microscopically in a Neubauer counting chamber at any given time.

\section{Results}

\section{Growth in Feinberg and Whittington medium}

Table 1 and Fig. 1 show the mean viable counts and the corresponding growth curves of 36 and 27 successive cultures of $T$. vaginalis strains $\mathrm{A}$ and $\mathrm{B}$, respectively. From these results it can be seen that both strains grew relatively well in Feinberg and Whittington medium. They both showed an equally early initiation of multiplication, but their growth rate was slow because it took $48 \mathrm{~h}$ to attain maximum growth with populations merely 10 -fold higher than those at the beginning of the experiment.

Growth curves of the two strains were similar, with well distinguished logarithmic, stationary and decline phases. However, during the logarithmic phase the growth rate of strain $\mathrm{A}$ was regular and progressive while that of strain B was temporarily arrested before recommencing, so that the corresponding part of its growth curve showed a biphasic pattern. Stationary and decline phases lasted $24 \mathrm{~h}$ and $72 \mathrm{~h}$, respectively. During the first $48 \mathrm{~h}$ of the decline phase the death rate of both strains was rather slow. After that time the death rate accelerated rapidly and no viable trichomonads were observed after incubation for $144 \mathrm{~h}$.
Estimation of mean generation time (MGT) and decimal reduction time (DRT) of strains $A$ and $B$ determined at corresponding points in their growth curves revealed that the former grew slightly faster and died, during the first part of its decline phase, more slowly than the latter (Table 2).

\section{Effect of inoculum size on growth and survival}

Figs. 2 and 3 present mean viable counts and the corresponding growth curves of the strain A and B cultures obtained by grouping the results for each strain separately on the basis of inoculum size. These results show that cultures of both strains raised with low density inocula, i.e., $<5.2 \times 10^{3} / \mathrm{ml}$, had a faster rate and a longer duration of growth and survival than those raised with inocula containing $\left(2.74 \times 10^{4}\right)$ $\left(5.21 \times 10^{4}\right)$ viable trichomonads. Inocula of $\left(7.8 \times 10^{3}\right)-\left(2.35 \times 10^{4}\right) / \mathrm{ml}$ gave intermediate figures for growth rate and duration of survival (data not shown). This inversely proportional relationship of inoculum density to growth rate and survival was more apparent with strain B. The estimated generation and decimal reduction times of both strains (Table 3) made clear the effect of inoculum size and the differences in growth characteristics between the two strains. Moreover, growth curves calculated on a smaller number of cultures of each strain, raised with low density inocula, were very similar to those calculated on the sum of all cultures.

\section{Effect of $\mathrm{pH}$ on growth and survival}

In the laboratory conditions used and in buffered and non-buffered media adjusted to $\mathrm{pH} 6.9$ the initiation of growth of strain A was retarded for at least $48 \mathrm{~h}$. After that time both cultures entered their logarithmic phase slowly, reached their peaks at $96 \mathrm{~h}$ and showed a similar pattern of decline; the $\mathrm{pH}$ of the buffered medium remained stable at $\mathrm{pH} 6.9$ while that of the unbuffered medium dropped progressively from 6.5 to $4.5 \mathrm{pH}$ units (Fig. 4a and b). However, $\mathrm{pH}$ values of 6.4-4.5 slightly favoured growth during the logarithmic and the early decline phases. These results showed that $\mathrm{pH}$ values in the range 6.0-6.5 inhibit the initiation of growth, prolonging the duration of the lag phase of $T$. vaginalis considerably.

Table 1. Growth and survival of $T$. vaginalis clinical strains A and B in Feinberg and Whittington medium

\begin{tabular}{|c|c|c|c|c|c|c|c|c|c|c|c|c|}
\hline \multirow[b]{2}{*}{ Strain } & \multirow{2}{*}{$\begin{array}{l}\text { Number } \\
\text { of growth } \\
\text { studies }\end{array}$} & \multicolumn{11}{|c|}{$\log _{10}$ mean viable counts of $T$. vaginalis after incubation for $(\mathrm{h})$} \\
\hline & & 0 & 2 & 4 & 6 & 8 & 24 & 48 & 72 & 96 & 120 & 144 \\
\hline A & 36 & 4.20 & 4.30 & 4.47 & 5.00 & 4.73 & 4.89 & 5.29 & 5.09 & 4.48 & 3.62 & - \\
\hline (SD) & & 2.13 & 2.05 & 3.44 & 2.51 & 4.48 & 2.95 & 3.17 & 3.04 & 2.68 & 1.97 & \\
\hline (SE) & & 1.35 & 0.46 & 0.77 & 0.56 & 1.00 & 2.18 & 2.40 & 2.26 & 1.90 & 1.19 & \\
\hline B & 27 & 4.30 & 4.70 & 4.72 & 4.98 & 4.96 & 4.98 & 5.30 & 5.20 & 4.20 & 3.17 & - \\
\hline (SD) & & 2.26 & 4.54 & 5.19 & 10.42 & 6.89 & 2.93 & 3.10 & 3.14 & 2.54 & 1.66 & \\
\hline (SE) & & 1.54 & 1.10 & 1.25 & 2.52 & 1.67 & 2.20 & 2.38 & 2.42 & 1.83 & 0.94 & \\
\hline
\end{tabular}




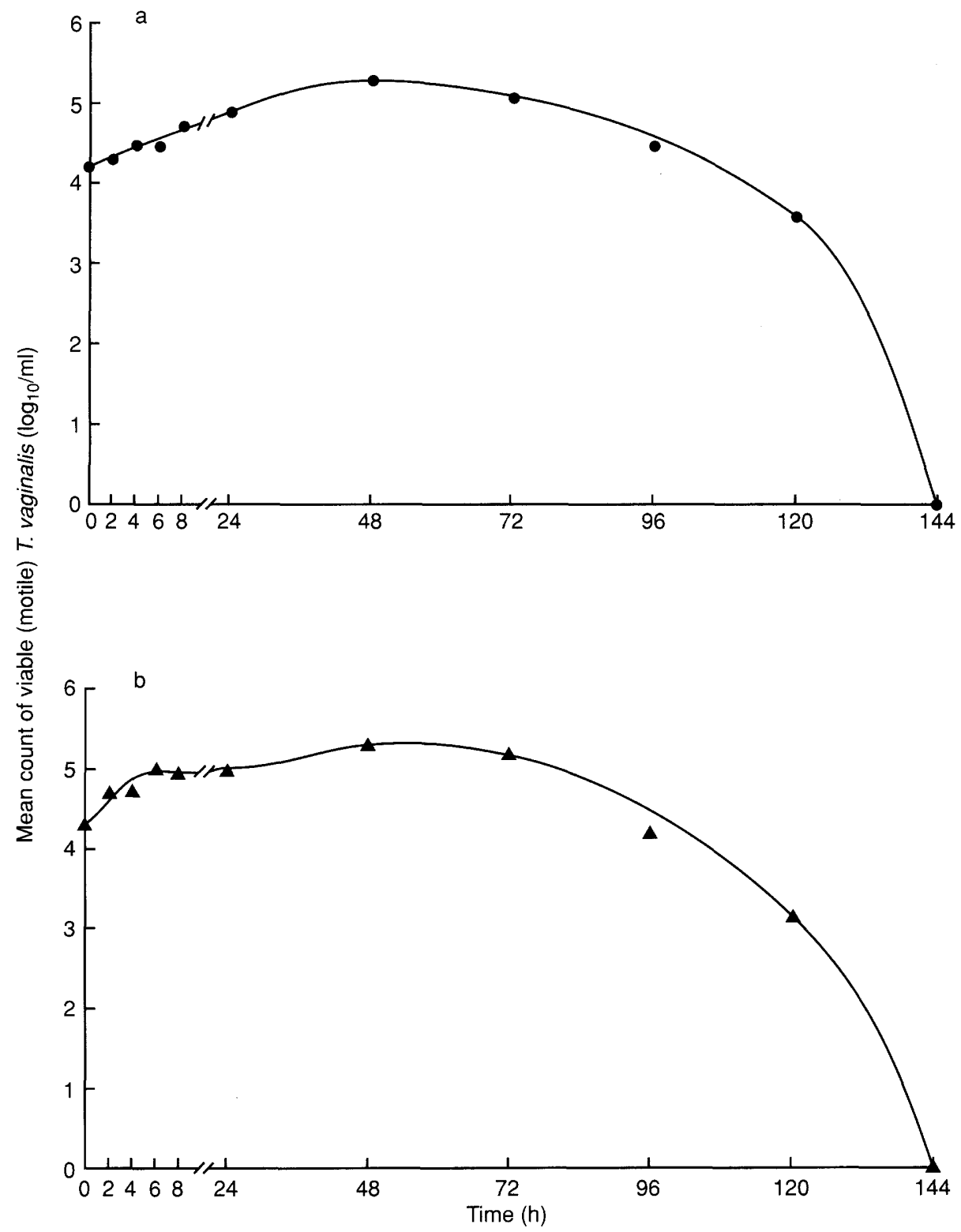

Fig. 1. Growth curves of (a) T. vaginalis strain A and (b). T. vaginalis strain B cultured anaerobically at $37^{\circ} \mathrm{C}$ in Feinberg and Whittington broth at $\mathrm{pH} 6.4$.

Table 2. Growth and death rate of $T$. vaginalis clinical strains $\mathrm{A}$ and $\mathrm{B}$ in Feinberg and Whittington medium

\begin{tabular}{lcccc}
\hline & & \multicolumn{3}{c}{ DRT between $\log _{10}$} \\
\cline { 3 - 5 } Strain & MGT & $5-4$ & $4-3$ & $3-2$ \\
\hline A & 11.5 & 31.6 & 17.2 & 6.7 \\
B & 14.4 & 25.5 & 16.8 & 9.6 \\
\hline
\end{tabular}

Mean generation time (MGT) and decimal reduction time (DRT) were estimated in hours.

\section{Effect of diluent on survival}

Normal saline and Ringer's solution proved deleterious for both strains of $T$. vaginalis. The lethal effect on $T$. vaginalis $5.02 \times 10^{4} / \mathrm{ml}$, suspended in either of the diluents, was progressive and started early after incubation for 15-30 min (Fig. 5a and b). Further incubation for 135-150 min caused an acute accelera- tion of death and the appearance of lysed protozoal cells in both diluents.

\section{Discussion}

This work revealed some important elements of the growth dynamics of the $T$. vaginalis strains tested and confirmed the relative suitability of Feinberg and Whittington medium for culturing and preservation purposes [6-9].

The early initiation of growth was unexpected and reflects the ability of the parasite to adjust to, and use without undue delay, a fresh nutritional environment devoid of toxic products of metabolism. The slow growth rate, the length of time needed for both strains to attain their rather low level of maximum growth 


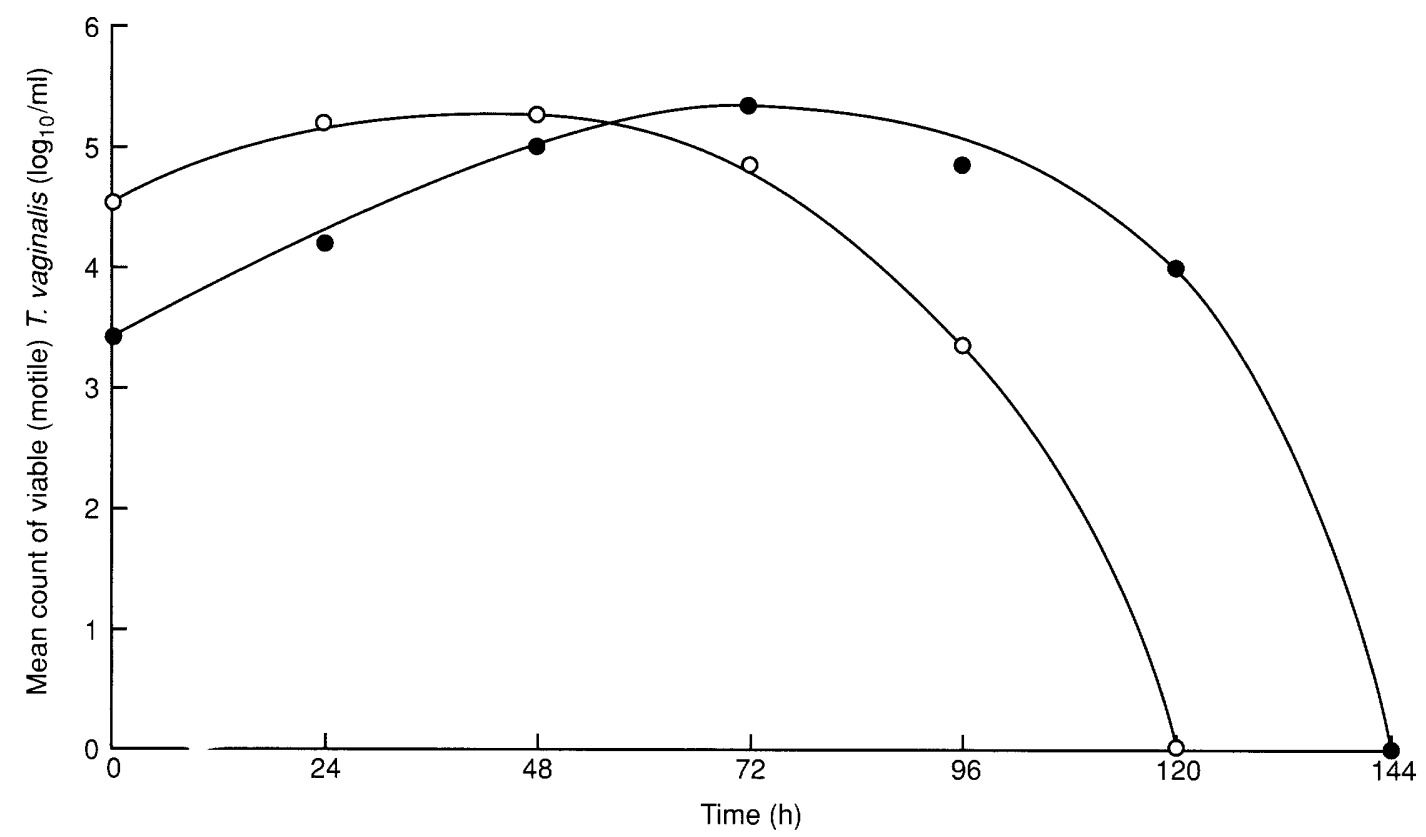

Fig. 2. Effect of inoculum density on viability of $T$. vaginalis strain A cultured anaerobically at $37^{\circ} \mathrm{C}$ in $\mathrm{Feinberg}$ and Whittington broth: 0 , low density inoculum; $O$, high density inoculum.

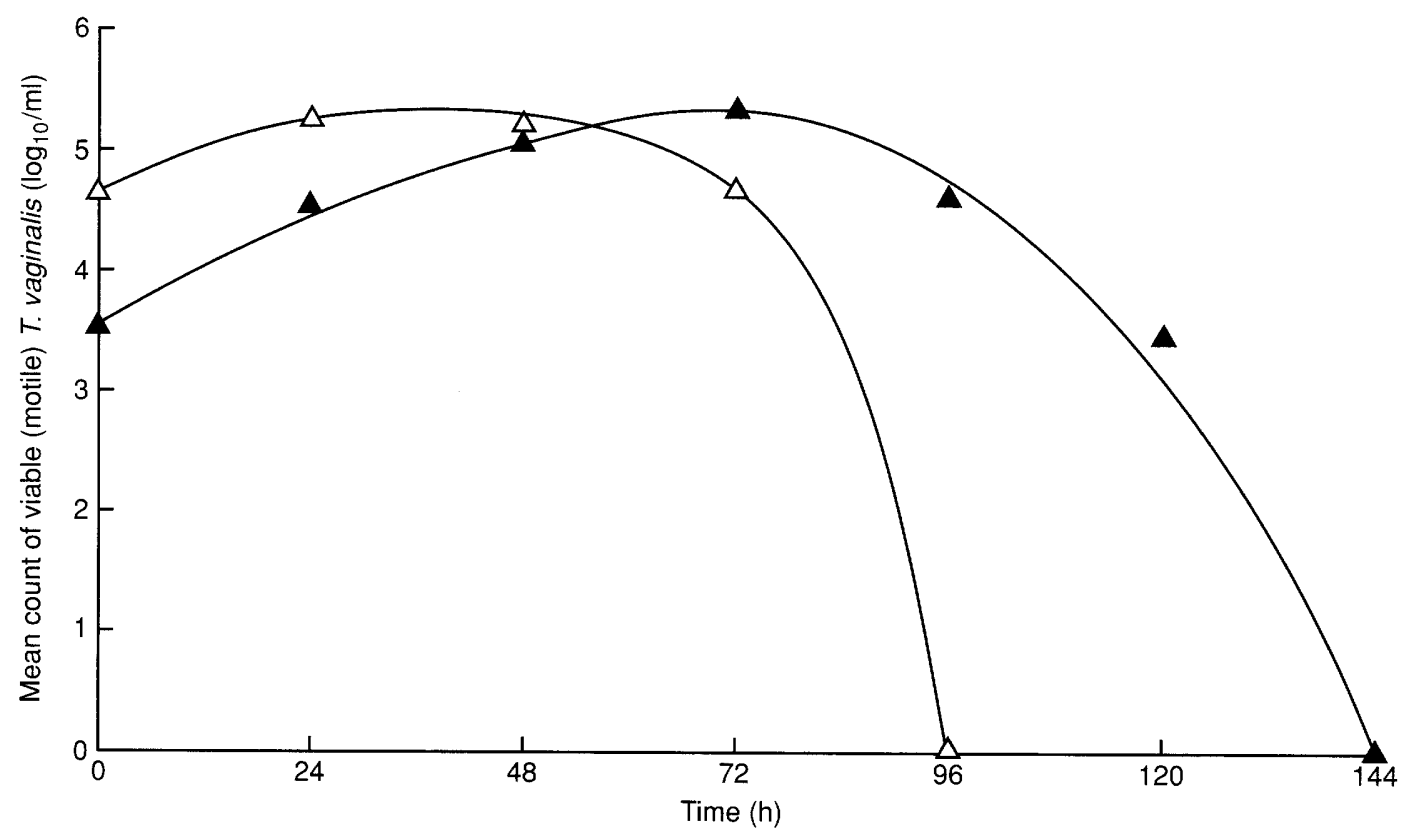

Fig. 3. Effect of inoculum density on viability of $T$. vaginalis strain $\mathrm{B}$ cultured anaerobically at $37^{\circ} \mathrm{C}$ in Feinberg and Whittington broth: $\boldsymbol{\Delta}$, low density inoculum; $\triangle$, high density inoculum.

Table 3. Growth and death rate of $T$. vaginalis clinical strains $\mathrm{A}$ and $\mathrm{B}$ in Feinberg and Whittington medium

\begin{tabular}{|c|c|c|c|c|c|c|c|c|}
\hline \multirow[b]{3}{*}{ Strain } & \multicolumn{4}{|c|}{ Low density inoculum } & \multicolumn{4}{|c|}{ High density inoculum } \\
\hline & \multirow[b]{2}{*}{ MGT } & \multicolumn{3}{|c|}{ DRT between $\log _{10}$} & \multirow[b]{2}{*}{ MGT } & \multicolumn{3}{|c|}{ DRT between $\log _{10}$} \\
\hline & & $5-4$ & $4-3$ & $3-2$ & & $5-4$ & $4-3$ & $3-2$ \\
\hline A & 9.4 & 25.4 & 11.0 & 5.3 & $\ldots$ & 20.2 & 13.4 & 8.6 \\
\hline B & 9.4 & 23.5 & 14.4 & 7.7 & & 19.7 & 7.5 & 3.8 \\
\hline
\end{tabular}

Mean generation time (MGT) and decimal reduction time (DRT) were estimated in hours; ..., estimation not possible. 

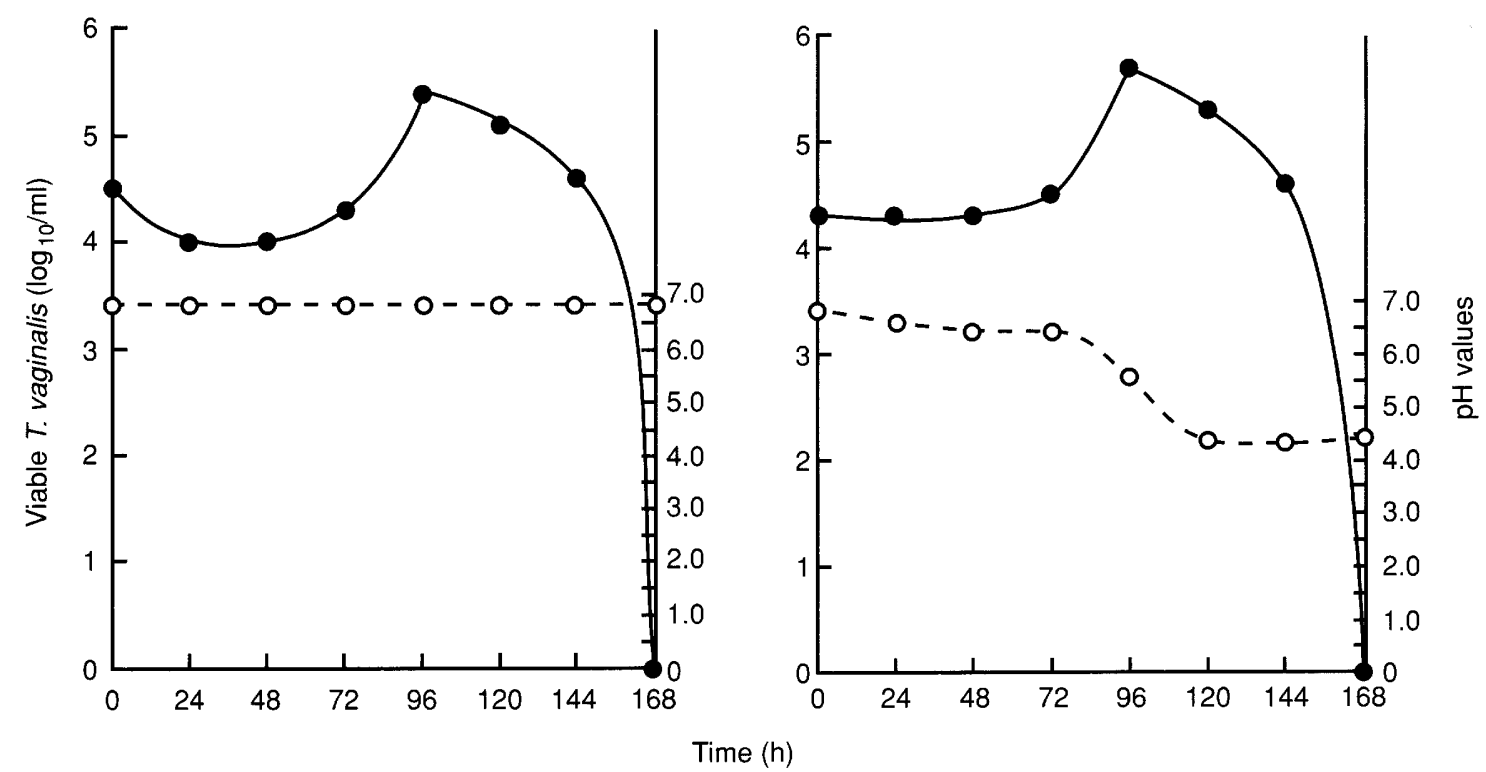

Fig. 4. (a) Effect of constant $\mathrm{pH} 6.9$ on viability of $T$. vaginalis strain A in buffered Feinberg and Whittington broth. (b) Effect of changing from $\mathrm{pH} 6.9$ to $\mathrm{pH} 4.5$ on viability of $T$. vaginalis strain A in non-buffered Feinberg and Whittington broth. 0 , mean viable count; $\mathrm{O}, \mathrm{pH}$ value.

a

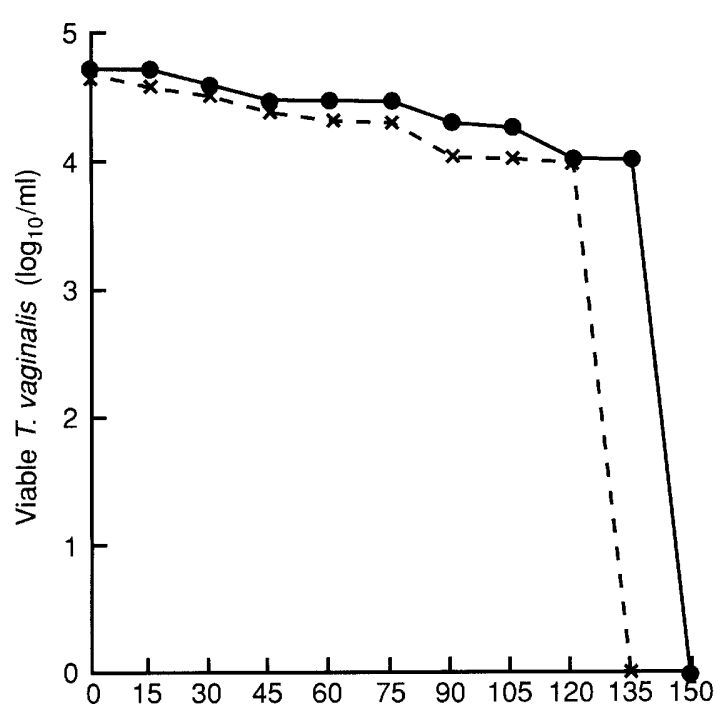

b

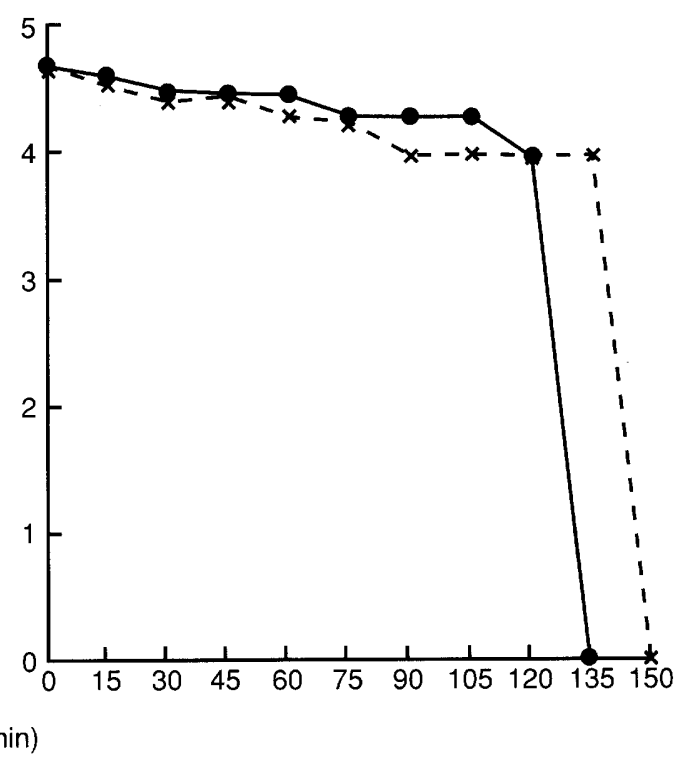

Fig. 5. Survival of $T$. vaginalis strains A (a) and B (b) in Ringer's solution both kept at $37^{\circ} \mathrm{C}$.

and the well-distinguished growth phases resemble the cultural features of slowly growing bacteria. On the other hand, the short duration of the decline phase and of survival are similar to those of highly exacting micro-organisms which are susceptible to the exhaustion of nutrients and the accumulation of toxic byproducts in the medium. However, the low density of population during all phases of growth and consequently the lack of a population effect [6] may partly contribute to the limited survival of $T$. vaginalis. These cultural features are in keeping with the clinical features and the progress of natural infection.
The population densities during various growth phases in serially repeated cultures and the growth curves constructed on the basis of the corresponding mean viable counts revealed growth similarities between the two strains. However, differences such as the biphasic logarithmic phase of strain B that makes doubtful the estimation of a mean generation time and its faster death rate are likely to be related to the existence of heterogeneity among strains of $T$. vaginalis. The variability in counts of motile trichomonads observed in repeated cultures of each particular strain further suggests the existence of inter-strain and intra-strain 
heterogeneity in multiplication rate. However, limitations of the counting method and the possible presence of more than one protozoal clone in each sample may also contribute to the variability of the viable counts.

The results of the effects of inoculum density on the growth of $T$. vaginalis in the present study are generally in agreement with earlier reports $[10,11]$. The inversely proportional relationship of inoculum size to growth rate and to duration of survival is a very complex phenomenon. The quicker exhaustion of nutrients and of other growth factors, the production and accumulation of greater amounts of inhibitory byproducts of metabolism by a denser growing population and the possible presence of more aged and consequently less active protozoa in larger inocula could partly explain these findings.

Results based on a smaller number of experiments involving high density inocula made the existing strain-dependent differences of the growth cycle between the two strains more apparent. Moreover, similarities between the growth curves of each strain based either on the sum of its cultures or on a smaller number of them, raised with low density inocula, confirm that the latter results are adequately reliable.

The effects of $\mathrm{pH}$ on the growth cycle of $T$. vaginalis are also very complex and it seems that a $\mathrm{pH}$ value of 6.4 is crucial for early initiation of growth whereas a $\mathrm{pH}$ of 6.9 retards it.

After the initiation of growth, $\mathrm{pH}$ values in the range 6.9-4.5 seem to have little influence because they affect the growth and the life-cycle of the parasite only slightly. The effects of $\mathrm{pH}$ on the growth of $T$. vaginalis in vitro are generally similar to the results of other studies and seem to be at least partly compatible with the clinical progress of the infection $[5,12]$. The duration of survival of light suspensions of $T$. vaginalis in both salt solutions tested at $37^{\circ} \mathrm{C}$ was very limited. Similar results, although not under exactly the same conditions, have been published earlier $[13,14]$. This initially progressive and finally destructive nature of the lethal effect of both diluents that is associated with lysis of the protozoal cells is difficult to explain. However, such findings might have been due to a progressive split of RNA [15, 16], degradation of rRNA $[15,17-19]$ and leakage of RNA-like material $[15,20]$ or proteins [20] that may occur in bacteria exposed to sublethal conditions in non-protective suspending media and they also give an indication of the possible anatomical or functional site of injury.
The in-vitro observations on the growth cycle of $T$. vaginalis in Feinberg and Whittington medium, on the effects of low density inocula and of $\mathrm{pH}$ and the lethal effect of the two diluents contribute to the in-vitro study of the life of the parasite. Moreover, they may prove useful in studies of the biology of this parasite and provide a growth model for other protozoa.

\section{References}

1. Rein MF, Chapel TA. Trichomoniasis, candidiasis, and the minor venereal diseases. Clin Obstet Gynecol 1975; 18: 73-88

2. McLellan R, Spence MR, Brockman M, Raffel L, Smith JL. The clinical diagnosis of trichomoniasis. Obstet Gynecol 1982; 60: $30-34$.

3. Honigberg BM. Trichomonas of importance in human medicine. In: Kreier JP (ed) Parasitic protozoa vol II: Intestinal flagellates, histomonads, trichomonads, amoeba opalimids, and ciliates. New York, Academic Press. 1978: 275-454.

4. Burch TA, Rees CW, Reardon LV. Epidemiological studies on human Trichomoniasis. Am J Trop Med Hyg 1959; 8: 312-318.

5. Gardner HL. Trichomoniasis. In: Gardner HL, Kaufman RH (eds) Benign diseases of the vulva and vagina, 2 nd edn. Boston, GK Hall. 1981: 243-271.

6. Feinberg JG, Whittington JM. A culture medium for Trichomonas vaginalis Donne and species of Candida. J Clin Pathol 1957; 10: 327-329.

7. Catterall RD. Trichomonal infections of the genital tract. Med Clin North Am 1972; 56: 1203-1209.

8. Balsdon MJ, Green N, Andrew CW, Jackson DH. Rapid staining technique for Trichomonas vaginalis. Preliminary report. Br J Vener Dis 1979; 55: 289-291.

9. Whittington MJ. Epidemiology of infections with Trichomonas vaginalis in the light of improved diagnostic methods. $\mathrm{Br} J$ Vener Dis 1957; 33: 80-91.

10. Kurnatowska A, Horwatt E. Quantitative study of Trichomonas vaginalis population in vitro with Candida albicans. Wiad Parazytol 1983; 29: 13-18.

11. Horwatt E. Analiza krzywych wzrostu Trichomonas vaginalis $\mathrm{w}$ obecnosci Staphylococcus aureus I Escherichia coli. [Analysis of growth curves of Trichomonas vaginalis in the presence of Staphylococcus aureus and Escherichia coli.] Wiad Parazytol 1985; 31: 113-123.

12. McGrory T, Meysick K, Lemchuk-Favel L, Garber GE. The interaction of Lactobacillus acidophilus and Trichomonas vaginalis in vitro. $J$ Parasitol 1994; 80: 50-54.

13. Postgate JR, Hunter JR. On the survival of frozen bacteria $J$ Gen Microbiol 1961; 26: 367-378.

14. Gorrill RH, McNeil EM. The effect of cold diluent on the viable count of Pseudomonas pyocyanea. J Gen Microbiol 1960; 22: 437-442.

15. Strange RE, Shon M. Effects of thermal stress on viability and ribonucleic acid of Aerobacter aerogenes in aqueous suspension. J Gen Microbiol 1964; 34: 99-114.

16. Iandolo JJ, Ordal ZJ. Repair of thermal injury of Staphylococcus aureus. J Bacteriol 1966; 91: 134-142.

17. Allwood MC, Russell AD. Mechanism of thermal injury in Staphylococcus aureus. I. Relationship between viability and leakage. Appl Microbiol 1967; 15: 1266-1269.

18. Nozawa R, Horiuchi T, Mizuno D. Degradation of ribosomal RNA in a temperature-sensitive Escherichia coli. Arch Biochem Biophys 1967; 118: 402-409.

19. Campbell LL, Pace B. Physiology of growth at high temperatures. J Appl Bacteriol II 1968; 31: 24-35.

20. Allwood MC, Russell AD. Thermally induced ribonucleic acid degradation and leakage of substances from the metabolic pool in Staphylococcus aureus. J Bacteriol 1968; 95: 345-349. 